Check for updates

Cite this: RSC Adv., 2018, 8, 13714

Received 20th February 2018

Accepted 27th March 2018

DOI: $10.1039 / c 8 r a 01542 b$

rsc.li/rsc-advances

\section{Sulfur compounds reactivity in the ODS of model and real feeds on W-SBA based catalysts $\uparrow$}

\author{
G. Estephane, (D) ${ }^{a}$ C. Lancelot, (D) *a P. Blanchard, ${ }^{a}$ J. Toufaily, ${ }^{b}$ T. Hamiye ${ }^{b}$ \\ and C. Lamonier iD a
}

W based catalysts were synthesized by dry impregnation of SBA-15 mesoporous silica with phosphotungstic acid (HPW) solution with W contents between 5 and 20\%, the HPW compound being preserved after calcination. The catalysts performance and the reactivity of various sulfide compounds were evaluated in the oxidative desulfurization (ODS) of model solutions and of real diesels, with sulfur contents ranging from 50 to $2000 \mathrm{ppm}$. The reactivity of benzothiophene and dibenzothiophene compounds was different in the ODS of model solutions but globally identical in the ODS of SRGO. The monitoring of the concentration of a range of alkyl DBT compounds (with alkyl groups from C2 to C5) in LGO confirmed the importance of the steric hindrance of alkyl substituents in the 4,6 position near the $S$ atom, as well as of the size of the alkyl groups. Among the $x$ W/SBA series, the catalyst with the highest loading showed the best performance in the ODS of LGO and SRGO while the catalysts efficiency could not be discriminated in the ODS of model solutions. In the ODS of both model solutions and real feeds, the W/ SBA catalyst was found to be much more efficient than a catalyst obtained by impregnation of a commercial silica with similar loading, highlighting the beneficial use of a mesoporous support with high surface area and pore volume that allowed well-dispersed tungsten species to be obtained. The quantity of sulfones precipitated and/or retained on the catalyst depended on the feed and was found to be higher in the ODS of model solution than in the ODS of real feeds. The precipitated/retained sulfones on the support may induce catalyst deactivation, which highlights the importance of the textural properties of the support. This detailed study points out the difficulty of extrapolating results obtained in the ODS of model solution to the ODS of real feeds.

\section{Introduction}

Environmental standards have been implemented to decrease the sulfur content of diesel fuel at very low levels, down to $10 \mathrm{ppm}$ in many countries, in order to reduce pollution of diesel engines. The tightening of sulfur specifications requires deep hydrodesulfurization (HDS) of diesel, a catalytic process generally performed on $\mathrm{CoMo} / \mathrm{Al}_{2} \mathrm{O}_{3}$ catalysts. However due to the alkyl groups close to the sulfur atom creating steric hindrance, some alkyl dibenzothiophene (DBT) compounds such as 4,6-DMDBT are known to be refractory in the conventional HDS process. ${ }^{1-3}$ The removal of these compounds by HDS to achieve the desired low levels of sulfur requires high temperature and $\mathrm{H}_{2}$ pressure conditions which increases the cost of the process. From an environmental and economic point of view, it is desirable to

${ }^{a}$ Université Lille, CNRS, ENSCL, Centrale Lille, Univ. Artois, UMR 8181 - UCCS - Unité de Catalyse et de Chimie du Solide, F-59000 Lille, France. E-mail: christine.lancelot@ univ-lille1.fr

${ }^{b}$ MCEMA, Lebanese University, Faculty of Science, Rafic Hariri University Campus, Hadath, Lebanon

$\dagger$ Electronic supplementary information (ESI) available. See DOI: 10.1039/c8ra01542b develop alternative, more energy efficient, desulfurization processes for the production of fuels with low sulfur content. Among alternative solutions for sulfur removal, oxidative desulfurization (ODS) appeared as particularly promising. In this process, sulfone species are formed from sulfide molecules and are further separated from the fuel with absorbents or by extraction using their polar properties. Indeed compared to conventional HDS, ODS can be carried out under mild temperature conditions, at atmospheric pressure and without the use of expensive hydrogen..$^{4-6}$

Also in favor of ODS, several studies have reported that the refractory molecules in HDS are the most reactive in ODS, in relation with the electron density on the sulfur atom: molecules with low electron density such as benzothiophene are less reactive than DBT and its derivatives. ${ }^{7-10}$ Comparing the reactivity of the alkyl-dibenzothiophenes, the desulfurization efficiency has been reported to decrease in the order: DBT > 4-MDBT > 4,6-DMDBT, thus mainly governed by the steric hindrance of the alkyl substituents near the sulfur atom. ${ }^{11-15}$ At the opposite, Sampanthar et al. observed that in their conditions of ODS of a model diesel, the oxidative reactivity followed the order: 4,6-DEDBT > 4,6-DMDBT > 4 -MDBT $>$ DBT, and that the increased electron density on the 
sulfur atom can compensate for the steric hindrance of the $\mathrm{C} 4$ and C6 alkyl groups. ${ }^{5}$

However, the majority of the papers dealing with sulfur molecules comparative reactivity in ODS refer to model solutions. Indeed, in real feeds, conversion is evaluated globally by measuring the sulfur content in the reaction medium after extraction of the formed sulfones. ${ }^{16,17}$ Only recently, Safa et al. reported the reactivity of alkyl dibenzothiophenes in the ODS of a hydrotreated middle distillate and found that it depends on the steric hindrance around the sulfur atom caused by the alkyl substituents at the 4- and 6-positions. ${ }^{18}$ Elwan et al. compared the efficiency of a bifunctional ionic liquid in the ODS of model oil (DBT $1000 \mathrm{ppmS}$ ), gasoline (500 ppmS) and diesel (12 $000 \mathrm{ppmS})$ and found a decrease of the global sulfur removal, attributed to more complex structure of the sulfur compounds as well as to the high sulfur content in the diesel. ${ }^{19}$ Precipitation of sulfones and their retention on the catalyst is scarcely mentioned, ${ }^{20}$ but may have an impact on the catalytic performance. Clearly the nature of the feed is of paramount importance and the reactivity of sulfur molecules determined in a simple medium may not be fully representative of that in much more complex feeds.

We thus propose in this work to study the ODS reaction of different feeds, with increasing complexity in terms of composition and sulfur content: model feeds, Light Gas Oil (LGO) and diluted Straight Run Gas Oil (SRGO), with sulfur content between 50 and 2000 ppmS. Moreover, efficiency of the catalysts was followed by gas chromatography equipped with a specific sulfur detector (GC-SCD) allowing to compare the reactivity of the different molecules or family of molecules depending on the matrix. The studied catalysts consisted in tungsten supported on SBA-15, obtained by impregnation of the carrier by phosphotungstic acid solution. Influence of the tungsten loading on the conversion was also considered, with $\mathrm{W}$ content varying between 5 and $20 \mathrm{wt} \%$ of $\mathrm{W}$.

\section{Experimental section}

\subsection{Support and catalysts synthesis}

The SBA-15 support was prepared under classical acidic conditions. ${ }^{21}$ Triblock copolymer $\mathrm{P} 123 \quad\left(\mathrm{EO}_{20} \mathrm{PO}_{70} \mathrm{EO}_{20}\right.$ rom Aldrich; $12.0 \mathrm{~g})$ was dissolved in water $(370 \mathrm{~g})$ acidified with $\mathrm{HCl}$ ( $37 \mathrm{~g}, 32 \mathrm{wt} \%$ ). The solution was then heated at $40{ }^{\circ} \mathrm{C}$. After the complete dissolution of the copolymer, tetraethyl orthosilicate (TEOS, 99\% from Fluka, $24 \mathrm{~g}$ ) was slowly added under vigorous stirring to give a gel with a TEOS/P123/ $\mathrm{HCl} / \mathrm{H}_{2} \mathrm{O}$ molar ratio of $1: 0.018: 3.3: 187$. The transparent solution was stirred at $40{ }^{\circ} \mathrm{C}$ for $24 \mathrm{~h}$. The obtained milky solution was then transferred to a Teflon lined autoclave and heated at $100{ }^{\circ} \mathrm{C}$ for $24 \mathrm{~h}$. After cooling down the autoclave to room temperature, the white solid was collected by filtration and washed with distilled water before drying at $80{ }^{\circ} \mathrm{C}$ overnight. Before characterization and subsequent use, the solid was calcined under air at $500{ }^{\circ} \mathrm{C}$ for $8 \mathrm{~h}\left(1^{\circ} \mathrm{C} \mathrm{min}^{-1}\right)$. The obtained solid presented a specific surface area of $1002 \mathrm{~m}^{2} \mathrm{~g}^{-1}$, a porous volume of $1.4 \mathrm{~cm}^{3} \mathrm{~g}^{-1}$ and an average pore diameter of $7.4 \mathrm{~nm}$.

The W-based catalysts were prepared by incipient wetness impregnation of a solution of phosphotungstic acid $\mathrm{H}_{3} \mathrm{PW}_{12} \mathrm{O}_{40}$
(HPW) on the SBA support with a W content from 5 to $20 \mathrm{wt} \%$, calculated by considering $\mathrm{WO}_{3}$ content in the calcined solid between 6 to $25 \mathrm{wt} \%$. After impregnation and maturation for $3 \mathrm{~h}$, the obtained solids were dried overnight in an oven at $75^{\circ} \mathrm{C}$ before calcination under air flow at $500{ }^{\circ} \mathrm{C}$ for $8 \mathrm{~h}$ with a temperature increase rate of $1^{\circ} \mathrm{C} \mathrm{min}^{-1}$.

For comparison purposes, a reference catalyst was prepared by impregnation of a commercial silica support with a $\mathrm{W}$ loading of $4 \mathrm{wt} \%$. The silica presented a surface area of $203 \mathrm{~m}^{2}$ $\mathrm{g}^{-1}$, a pore volume of $0.9 \mathrm{~cm}^{3} \mathrm{~g}^{-1}$ and a mean pore diameter of $15 \mathrm{~nm}$ (broad distribution between 5 and $30 \mathrm{~nm}$ ).

The prepared catalysts were named $x \mathrm{~W} / \mathrm{SBA}$, in which $x$ is the $\mathrm{W}$ content (wt\%). Similarly the reference sample was denoted $4 \mathrm{~W} / \mathrm{SiO}_{2}$.

\subsection{Solids characterization}

$\mathrm{N}_{2}$ adsorption-desorption isotherms were recorded at $-196{ }^{\circ} \mathrm{C}$ using an automated ASAP2010 instrument from MICROMERITICS. A known mass of sample (around $0.200 \mathrm{~g}$ ) was heated at $350{ }^{\circ} \mathrm{C}$ under vacuum for $3 \mathrm{~h}$. Specific surface areas (SSA) were calculated from the linear part of the BrunauerEmmett-Teller curve. Pore size distributions were obtained by applying the Barrett-Joyner-Halenda (BJH) equation to the desorption branch of the isotherm and total pore volume was estimated from the $\mathrm{N}_{2}$ uptake value at $P / P_{0}=0.98$. W species present on the support were identified by Raman spectroscopy. Spectra of the oxidic precursors were recorded at room temperature, using a Raman microprobe infinity instrument from Jobin-Yvon, equipped with a $\mathrm{N}_{2}$ cooled CCD detector. The exciting laser source was the $532 \mathrm{~nm}$ line of a Nd-YAG laser.

\subsection{Catalytic evaluation in ODS reaction}

Catalysts performance were evaluated in the ODS of model and real feedstocks, the sulfur content varying between 50 and 2000 ppms: (i) a model solution of dibenzothiophene (DBT) in dodecane with concentrations of 50 and 500 ppmS named M50 and M500 respectively; (ii) a mixed model solution of methylbenzothiophene (C1-BT), DBT and 4,6-dimethyldibenzothiophene (4,6-DMDBT) in dodecane with concentrations of 600 and 1500 ppmS named MM600 and MM1500 respectively, which were obtained by mixing 200 and $500 \mathrm{ppm}$ of each compound; (iii) a light gas oil with 50 ppmS named LGO50; (iv) a straight run gas oil with 2000 ppmS named SRGO2000 (obtained by dilution of a $1 \%$ S SRGO with LGO50).

The oxidation reaction was performed in a $200 \mathrm{~mL}$ batch reactor under reflux. The solution to be desulfurized was first loaded in the reactor and heated to $75{ }^{\circ} \mathrm{C}$. Catalyst and oxidant (tert-butyl hydroperoxide, TBHP) were added simultaneously to the reaction mixture under stirring at $700 \mathrm{rpm}$, the ratios mass(catalyst)/mass(solution) being set at 0.01 and 0.02 and the oxidant/S ratio at 2.3 and 25 for model and real charges, respectively. Indeed $\mathrm{O} / \mathrm{S}$ ratios only slightly higher than the stoichiometric one (equal to 2) are required in the ODS of model fuels ${ }^{22-25}$ while much larger values (above 15) are reported in the literature when real feeds are treated. ${ }^{6,18}$ These high values are justified by possible secondary oxidation reactions of aromatics 
or nitrogen compounds present in these feeds. At the end of the test, the reaction medium was separated by filtration and the collected solid was washed with pentane to remove DBT and dodecane, and with methanol that due to its polar properties solubilized the sulfones precipitated or retained on the catalyst.

Reaction medium and washing solutions were analyzed by UV fluorescence on an Antek 9000 apparatus to determine the total sulfur content and by gas phase chromatography on a Varian 3800 equipped with a sulfur specific detector Sievers SCD 355 to identify and evaluate the quantity of sulfur containing compounds. These analyses allowed calculating the conversion of the sulfide molecules and the retention rate of sulfones. The retention rate is calculated as the difference of the sulfur content in the initial solution and in the reaction solution after filtration and corresponds to sulfones precipitated and/or retained on the catalyst. Analysis of the washing solutions by chromatography confirmed the presence of only sulfones. Sulfur balances were found in all cases close to $100 \%$.

In model solutions, the conversion of sulfide species was obtained by dividing the mass of formed sulfones by the initial mass of sulfur compounds (Formula 1). The mass of sulfones is deduced from the quantity of sulfones present in the reaction mixture and in the washing solutions of the catalyst (Formula 2, $2^{\prime}$ and $2^{\prime \prime}$ ) and is calculated considering the global amount of sulfur in the solutions as determined by UV fluorescence and the relative intensities of the chromatogram peaks of sulfides and sulfones present in these solutions (formula 3). For these calculations we have considered that the response factors of the different sulfur containing molecules (sulfides and sulfones, with alkyls groups or not) are similar since each type of molecule contains only one sulfur atom.

$$
\text { Conv. }=\operatorname{mass}(\text { sulfones }) / \operatorname{mass}\left(S_{\text {tot }}\right)
$$

$$
\text { Mass(sulfones) } \left.=\text { mass(sulfones) })_{\text {reac }}+\text { mass(sulfones) }\right)_{\text {wash }}
$$

Mass(sulfones) $)_{\text {reac }}=\operatorname{mass}($ sulfones $)$ in the reaction medium $\left(2^{\prime}\right)$

Mass(sulfones) $)_{\text {wash }}=\operatorname{mass}($ sulfones $)$ in the washing solution $\left(2^{\prime \prime}\right)$

Mass(sulfones) in washing or reaction solution $=$

$$
\left(A_{\text {sulfones }} /\left(A_{\text {sulfides }}+A_{\text {sulfones }}\right)\right) \times S_{\text {tot }}
$$

$A_{\mathrm{j}}$ being the area of the peak of the compound $\mathrm{j}$ (sulfones or sulfides) in the chromatograms, $S_{\text {tot }}$ being the total sulfur content of the solution as determined by UV fluorescence.

In LGO and SRGO feeds, considering the complexity of the chromatograms of the feed and of the reaction medium, only conversion of some individual species or families of compounds could be followed by integration of the corresponding peaks in the chromatograms.

\section{Results and discussion}

\subsection{Catalysts characterization}

Raman spectroscopy analysis was performed on the calcined solids (Fig. 1). The broad lines pointed at 780 and $1090 \mathrm{~cm}^{-1}$ correspond to the SBA support. On all catalysts, the

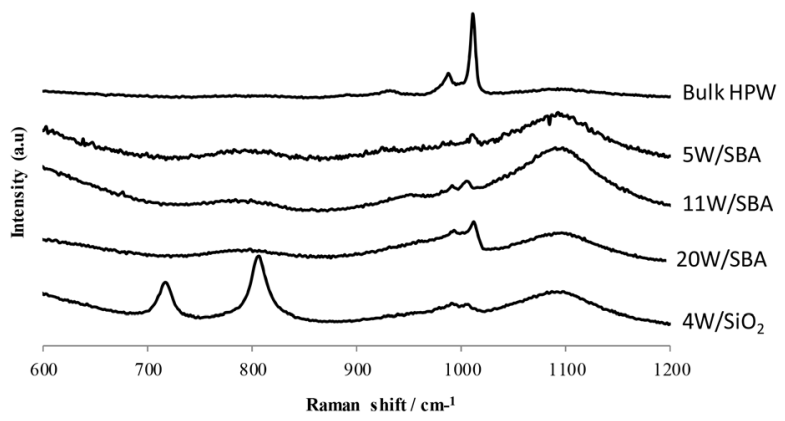

Fig. 1 Raman spectra of bulk HPW, calcined $x / \mathrm{SBA}$ and $4 \mathrm{~W} / \mathrm{SiO}_{2}$.

characteristic lines of HPW have been identified at 1009, 992 and $930 \mathrm{~cm}^{-1}$, indicating that the HPA is still present after calcination. ${ }^{26}$

Such phenomenon was already observed by Thouvenot et $a .^{27}$ and Rocchiccioli-Deltcheff et $a .^{28}$ who reported the presence of $\mathrm{H}_{4} \mathrm{PMO}_{12} \mathrm{O}_{40}$ after calcination at $500{ }^{\circ} \mathrm{C}$ of $\mathrm{H}_{4} \mathrm{PMo}_{12} \mathrm{O}_{40} / \mathrm{SiO}_{2}$ type catalysts. Thouvenot et al. showed that this heteropolyacid was effectively decomposed during calcination, giving $\mathrm{SiO}_{2}$ and $\mathrm{MoO}_{3}$, and reformed at room temperature under water vapor pressure at the surface of the silica through the following reaction: $\mathrm{SiO}_{2}+12 \mathrm{MoO}_{3}+2 \mathrm{H}_{2} \mathrm{O}=$ $\mathrm{H}_{4} \mathrm{PMo}_{12} \mathrm{O}_{40}$. Rocchiccioli-Deltcheff et al. have proposed that this reaction can be considered as a reaction in aqueous solution occurring in the presence of water in the porosity of silica. Concerning our catalyst, such a phenomenon could be considered to explain the presence of HPW in the SBA porosity. Indeed after calcination the solid is stored at room temperature under ambient air and thus re-adsorbs probably significant amount of water that could permit the reformation of HPW via a similar reaction, even if preservation of the HPW cannot be ruled out.

On the $4 \mathrm{~W} / \mathrm{SiO}_{2}$ catalyst on commercial $\mathrm{SiO}_{2}$ support, lines at 716 and $805 \mathrm{~cm}^{-1}$ evidencing the presence of $\mathrm{WO}_{3}$ were identified (Fig. 1). No re-formation of HPW after air exposure is thus observed. Similarly, bulk HPW was calcined at $500{ }^{\circ} \mathrm{C}$ and the Raman spectra (not shown here) attested the presence of $\mathrm{WO}_{3}$. We can propose that (i) on $\mathrm{SiO}_{2}$, the HPW before calcination is not in a well-dispersed state, and thus behaves in a similar way than bulk HPA, with $\mathrm{WO}_{3}$ formation during calcination; (ii) on SBA, the HPW is well-dispersed and in interaction with the support that could maintain the HPW throughout calcination or at least prevent its decomposition in $\mathrm{WO}_{3}$ that seems unlikely to re-form HPW after air exposure, as seen from our observations. This highlights the beneficial use of SBA with improved textural properties as a support to obtain a well-dispersed tungsten phase.

\subsection{ODS of a model solution of DBT}

A first set of ODS tests was performed on solutions of DBT in dodecane with two sulfur contents: $50 \mathrm{ppmS}$ (M50) and 500 ppmS (M500). 
On M50, blank experiments were carried out (i) without catalyst and (ii) on the bare support, with respective conversions after one hour of 10 and $13 \%$.

The conversion of sulfides on $x \mathrm{~W} / \mathrm{SBA}$ solids obtained in the ODS of M50 after one hour of reaction are presented in Table 1. For all solids high conversions of DBT were obtained, between 93 and $100 \%$. Composition of the reaction medium showed very low global sulfur content, between 2 and 5 ppm, consisting of $\mathrm{DBTO}_{2}$ and DBT when not fully converted. Analysis of the washing solution revealed exclusively the presence of sulfones, precipitated in the reaction medium or deposited on the catalyst, with high retention rates around 95\%.

Catalysts 5W/SBA, 11W/SBA and 20W/SBA were further evaluated in more severe conditions, in the ODS of a solution of DBT at $500 \mathrm{ppms}$ in a simulation of dynamic test with addition of 500 ppmS after one and two hours of reaction (keeping the same $\mathrm{O} / \mathrm{S}$ ratio). Table 1 shows the conversions after one hour of reaction (on 500 ppmS DBT), one hour after the first addition of 500 ppms (globally on 1000 ppmS DBT) and one hour after the second addition (globally on $1500 \mathrm{ppms}$ ). After one hour of oxidation, $100 \%$ of DBT was converted on all solids, which is equivalent to their behavior on M50. One hour after the first addition of 500 ppmS, a small quantity of unconverted DBT appeared in the reaction medium, the conversion remaining however between 95 and 98\%. After the second addition the conversion decreased slightly from 95 to $91 \%$ on $5 \mathrm{~W} / \mathrm{SBA}$ while on $11 \mathrm{~W} / \mathrm{SBA}$ and $20 \mathrm{~W} / \mathrm{SBA}$, similar and very high conversions around $98 \%$ were maintained. Increasing $\mathrm{W}$ content from 5 to $11 \%$ is thus slightly beneficial to the performance, while further added tungsten do not contribute to improving the activity.

High conversions in the ODS of DBT 500 ppms have also been reported in the literature. Abdalla et al. claimed a conversion of $93 \%$ after one hour of reaction on a catalyst based on $\mathrm{W}$ and $\mathrm{Al}_{2} \mathrm{O}_{3}\left(13 \% \mathrm{WO}_{3}\right) .{ }^{29}$ Xie et al. observed on a solid $\mathrm{W}$ on MCM-41 (6\% $\left.\mathrm{WO}_{3}\right)$ a total conversion of DBT after $180 \mathrm{~min}$ of reaction. ${ }^{30}$ However, the same catalyst appeared less efficient on a model solution of $800 \mathrm{ppmS}$ of DBT, with only $60 \%$ after 30 min of reaction and $80 \%$ at one hour. According to Qi et al., catalysts based on HPW supported on Zr-SBA converted 58, 95 and 97\% of DBT 1000 ppmS with $\mathrm{WO}_{3}$ loadings of 10, 19 and $29 \% \mathrm{WO}_{3}$ respectively. ${ }^{31}$

In comparison, the conversion of impregnated solid on commercial silica $4 \mathrm{~W} / \mathrm{SiO}_{2}$ is much lower with values around $40 \%$ whatever the sulfur content of the solution to be treated. Indeed Raman analysis evidenced the presence of $\mathrm{WO}_{3}$ on $\mathrm{SiO}_{2}$ support, which appears to be detrimental to ODS activity compared to well-dispersed HPW, observed on SBA.

Table 1 Conversion of DBT in the ODS of M50 and M500 on $x$ W/SBA

\begin{tabular}{lllll}
\hline & $\begin{array}{l}\text { Conv. } \\
\text { M50/\% }\end{array}$ & $\begin{array}{l}\text { Conv. } \\
\text { M500 (1)/\% }\end{array}$ & $\begin{array}{l}\text { Conv. } \\
\text { M500 (2)/\% }\end{array}$ & $\begin{array}{l}\text { Conv. } \\
\text { M500 (3) } / \%\end{array}$ \\
\hline 5W/SBA & 100 & 100 & 95 & 91 \\
14W/SBA & 94 & 100 & 97 & 98 \\
20W/SBA & 93 & 100 & 98 & 97
\end{tabular}

Textural analysis were conducted on catalyst 20W/SBA after test, after two pretreatments: (i) washing with pentane, which eliminates DBT and dodecane residues present on the catalyst (named 20W/SBA_P) and (ii) washing with methanol to remove any adsorbed sulfones (named 20W/SBA_M). The treated solids were then dried at room temperature and degassed at $150{ }^{\circ} \mathrm{C}$ for 6 hours. Specific area $\left(773 \mathrm{~m}^{2} \mathrm{~g}^{-1}\right)$ and pore volume $\left(1.0 \mathrm{~cm}^{3}\right.$ $\mathrm{g}^{-1}$ ) of the catalyst after test washed with pentane 20W/SBA_P were found lower than those of the fresh catalyst $\left(972 \mathrm{~m}^{2} \mathrm{~g}^{-1}\right.$ and $1.6 \mathrm{~cm}^{3} \mathrm{~g}^{-1}$ ), while the porous distribution was not modified evidencing pore plugging (Fig. SI $\dagger$ ). Washing with methanol, which dissolves sulfones, allowed to partly recover the initial specific surface area $\left(816 \mathrm{~m}^{2} \mathrm{~g}^{-1}\right)$ and pore volume $(1.3$ $\left.\mathrm{cm}^{3} \mathrm{~g}^{-1}\right)$. These results indicate sulfones deposition in the catalyst pores, occurring during ODS reaction. The textural properties of the support are thus an important parameter regarding the performance and lifetime of the catalysts.

\subsection{ODS of a mixed model solution of BT, DBT and 4,6- DMDBT}

The catalyst $5 \mathrm{~W} / \mathrm{SBA}$ was further evaluated in the ODS of a mixed solution of BT, DBT and 4,6-DMDBT with $600 \mathrm{ppmS}$ (MM600) and 1500 ppms (MM1500).

The chromatograms of the reaction mixture during the first two hours of reaction are reported in Fig. 2 and the concentrations of the three sulfur species present in solution during the first hour of reaction in Fig. 3. At 5 min of reaction, an important decrease in the intensity of the peaks corresponding to DBT and 4,6-DMDBT is
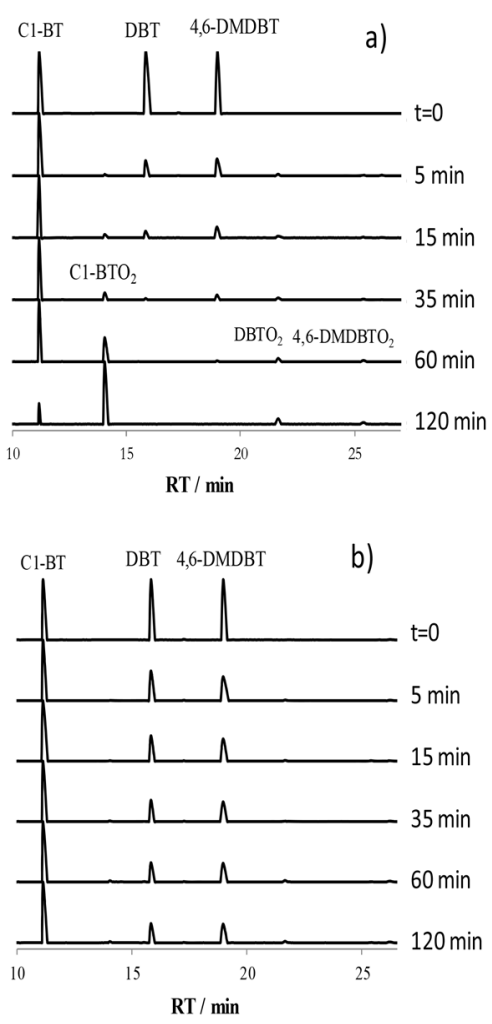

Fig. 2 Chromatograms of the reaction mixture in the ODS of MM600 (a) and MM1500 (b) on 5W/SBA between 0 and $2 \mathrm{~h}$. 


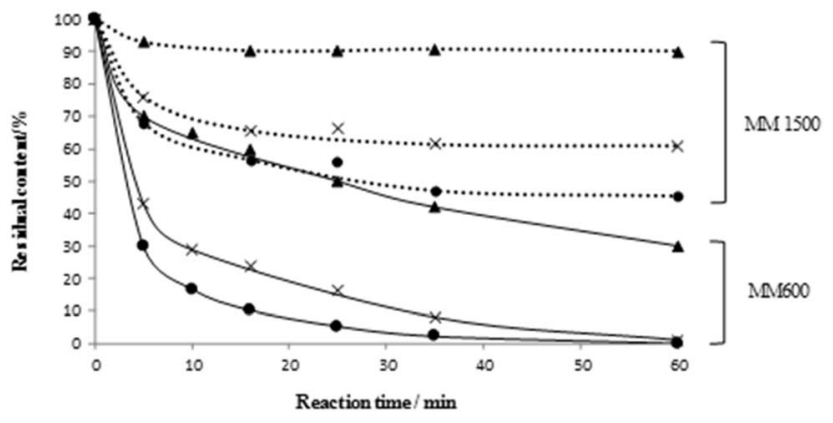

Fig. 3 Percentages of residual concentrations of C1-BT ( $\mathbf{\Delta}), 4,6-$ $\operatorname{DMDBT}(x)$ and DBT $(-)$ during the ODS of MM600 (-) and MM1500 $(\cdots)$ on $5 \mathrm{~W} / \mathrm{SBA}$

noticed on MM600, while the C1-BT appeared little affected. At 35 min, almost all DBT and 4,6-DMDBT have reacted, while 55\% of unreacted C1-BT were still present in solution. Very few sulfones were present in the reaction mixture. At $1 \mathrm{~h}$, one can still note the persistent presence of C1-BT, with $30 \%$ of unreacted species. The corresponding sulfone, $\mathrm{C} 1-\mathrm{BTO}_{2}$, is mostly present in the reaction medium, in contrast to other sulfones.

Similar results were reported in the literature. Wang et al. obtained conversions of $45 \%$ for BT and between 80 and $85 \%$ for DBT, 4-DMDBT and 4,6-DMDBT, in the ODS of a model solution $(10 \mathrm{ppmS})$ with $\mathrm{TBHP} / \mathrm{S}=3$ on a $16 \% \mathrm{MoO}_{3} / \mathrm{Al}_{2} \mathrm{O}_{3}$ catalyst. ${ }^{\mathbf{1 4}}$ On ordered meso-macro phosphotungstic acid HPW/ $\mathrm{SiO}_{2}(20 \% \mathrm{HPW})$ in the ODS of a model solution (250 ppmS), sulfur removal of BT, DBT, 4-MDBT and 4,6-DMDBT were 83\%, 99,89 and $86 \%$ respectively, after 40 min of reaction with $\mathrm{H}_{2} \mathrm{O}_{2}$ / $\mathrm{S}$ ratio of $4 .^{12}$

On MM1500, at 35 min of reaction, only $50 \%$ of DBT, $35 \%$ of 4,6-DMDBT and $10 \%$ of C1-BT have reacted, with no noticeable evolution of the concentration afterwards. Compared with MM600, increasing the sulfur content of the model feed led to lower conversion of the sulfide species. Indeed the mass(catalyst)/mass(charge) was kept constant (0.1) which implies a molar ratio W/S of 0.14 and 0.05 in MM600 and MM1500 respectively. Less tungsten atoms were thus available for sulfide molecules conversion in MM1500. Moreover, all formed sulfones were precipitated and/or retained on the catalyst, which associated to the low $\mathrm{W} / \mathrm{S}$ ratio, can lead to deactivation of the catalyst during the oxidation of MM1500.

Regarding the respective reactivities of the sulfide species, both in MM600 and MM1500, the order of reactivity is DBT > 4,6-DMDBT $\gg$ C1-BT (Fig. 3). C1-BT appeared to be less reactive than the DBT compounds, in relation with its lower electronic density on the sulfur atom (5.739 for BT and 5.758 for DBT). ${ }^{7-10}$ The electronic density of 4,6 -DMDBT is only slightly higher than that of DBT (5.760) and in this case the steric hindrance of the methyl groups near the sulfur atom governs the reactivity, with higher conversion of DBT compared to 4,6DMDBT. ${ }^{11-15}$

\subsection{Oxidation of industrial LGO}

The catalysts were tested in the oxidation of a hydrotreated gas oil, a light gas oil with $50 \mathrm{ppm}$ of sulfur (LGO50), thus containing only the most refractory compounds to HDS, alkyldibenzothiophenes. ODS of LGO50 was followed during one hour of test, with samplings carried out at 3, 15, 25, 35 and $60 \mathrm{~min}$ (Fig. $4 \mathrm{a}$ and $\mathrm{b}$ for 5W/SBA and 20W/SBA, Fig. SI $\uparrow \uparrow$ for $11 \mathrm{~W} / \mathrm{SBA})$. In the case of $5 \mathrm{~W} / \mathrm{SBA}$, a gradual decrease in the intensities of the peaks of sulfides is observed, with in parallel emergence of sulfones peaks. Increasing the $\mathrm{W}$ loading led to an increase of the performance, with for $11 \mathrm{~W} / \mathrm{SBA}$ and $20 \mathrm{~W} / \mathrm{SBA}$ most of the species being transformed after 3 minutes. These catalysts appear to be very efficient compared to those studied in the literature. Safa et al. followed by chromatography the ODS reaction of a hydrotreated middle distillate $(340 \mathrm{ppmS})$ with cumene peroxide as oxidizing agent $(\mathrm{O} / \mathrm{S}=20)$ on a $12 \mathrm{wt} \%$ $\mathrm{MoO}_{3} / \mathrm{Al}_{2} \mathrm{O}_{3}$ catalyst and observed conversions of the alkyl-DBTs between 70 and $90 \%$ after $1 \mathrm{~h}$ of reaction. ${ }^{18}$ Ishihara et al. in the ODS of a LGO with $39 \mathrm{ppmS}$ on a $16 \% \mathrm{wt} \mathrm{MoO}_{3} / \mathrm{Al}_{2} \mathrm{O}_{3}$ catalyst, with a TBHP/S ratio of 15 , found conversions of alkyl-DBTs between 72 and $87 \%$ after 3 h of reaction. ${ }^{6}$

Analysis of the washing solution after test confirmed the presence of only sulfones in the washing solutions, with retention rates around $50 \%$ for all solids, to be compared with 95\% after ODS of M50, containing only DBT with the same sulfur content than LGO50. The matrix of LGO50 appears more favourable to sulfones dissolution than the model solution in dodecane, taking into account the properties of the feed as well as the lower content of each molecule. Less deactivation due to sulfone deposition on the catalysts is thus to be expected.

The concentrations of 6 sulfide molecules were monitored during the first $5 \mathrm{~min}$ of the ODS test in order to compare the reactivity of molecules representative of the different alkyl-DBTs
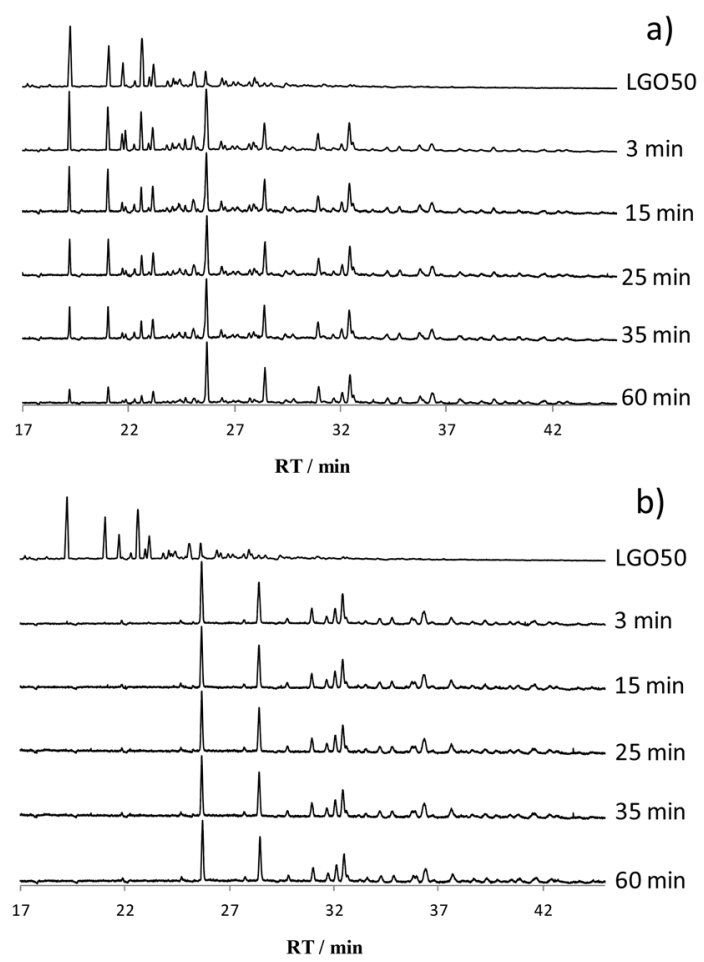

Fig. 4 Chromatograms of the reaction mixture in the ODS of LGO50 on 5W/SBA (a) and 20W/SBA (b) 
compounds: 4,6-DMDBT, 4-ethyl,6-methyl dibenzothiophene (4-E,6-MDBT), two trimethyl dibenzothiophenes TMDBT-1, TMDBT-2, 4,6-diethyl dibenzothiophene (4,6-DEDBT) and one alkyl DBT with 5C in the substituents (C5DBT-1), with respective retention times of 19.2, 21.0, 21.7, 22.6, 23.1 and $25.1 \mathrm{~min}$ (Fig. SI $3 \dagger$ ). In this region of the chromatograms only sulfide species are present, as the lowest retention time attributed to a sulfone peak $\left(4,6-\mathrm{DMDBTO}_{2}\right)$ is $25.6 \mathrm{~min}$ (as identified in the ODS of mixed model solution). This ensures that the integration of the peaks of sulfide molecules will not be disrupted by the presence of sulfones.

In the ODS of LGO50 on 5W/SBA, alkyl-DBTs reactivity increases in the following order: 4,6-DEDBT $<4$ E6MDBT $\approx \mathrm{C} 5$ DBT-1 < 4,6-DMDBT < TMDBT-1 < TMDBT-2 (Fig. 5a), which shows that it depends on the steric hindrance as well as the number of methyl substituents. Indeed 4,6-DEDBT (C4 group), 4-E,6-MDBT (C3 group) and 4,6-DMDBT (C2 group) with alkyl substituents in the same 4,6 position near the $S$ atom are the most refractory molecules and their reactivity increases when the number of substituting $\mathrm{C}$ decreases. The TMDBT- 1 and TMDBT-2 are the most reactive of the series, they are more reactive than the other $\mathrm{C} 3$ compound 4-E,6-MDBT and even than the $\mathrm{C} 2$ compound with a maximum steric hindrance towards the S atom, 4,6-DMDBT. Similarly the C5-DBT-1 is more reactive than the $\mathrm{C} 4$ 4,6-DEDBT. Very few papers address this issue in real feeds. Safa et al. studied the reactivity of various alkyl DBTs in the ODS of middle distillate and reported that 4E,6-MDBT was the less reactive of C3-DBT compounds, indicating that the steric size of the alkyls substituents in 4 and 6positions influences the reactivity. ${ }^{18}$

At higher $\mathrm{W}$ loadings, the reactivity of the all sulfide species increased while the difference between their behaviours became less pronounced. Finally, on the most efficient catalyst
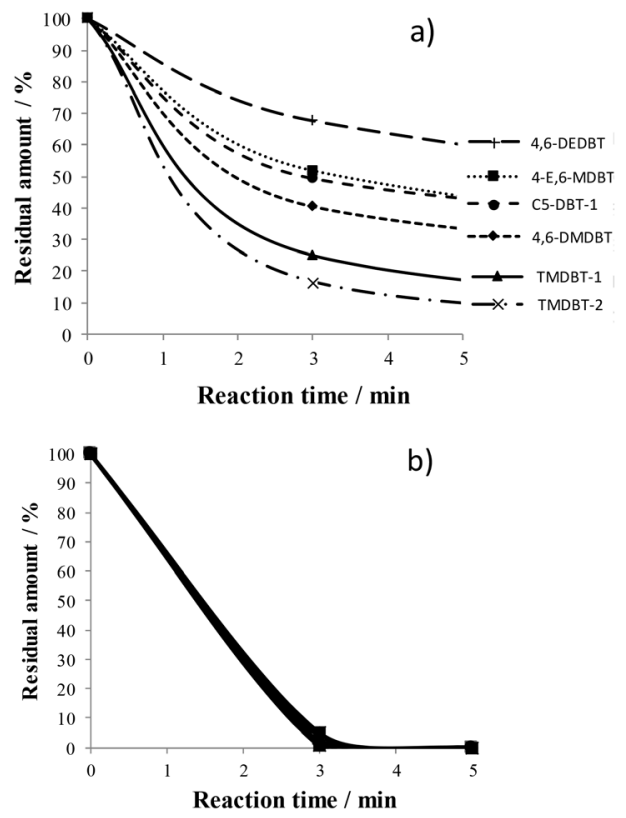

Fig. 5 Percentages of residual concentrations of the 6 selected species in the ODS of LGO50 on 5W/SBA (a) and 20W/SBA (b).
20W/SBA, the six sulfide species were simultaneously converted and almost completely oxidized after $3 \mathrm{~min}$ of reaction (Fig. 5b). $11 \mathrm{~W} / \mathrm{SBA}$ presented an intermediate behaviour, however closer to that of $20 \mathrm{~W} / \mathrm{SBA}$ (Fig. SI $4 \dagger$ ).

In the ODS of MM500, the influence of the $\mathrm{W}$ content was similar to that observed in the ODS of LGO50, with the lowest conversion obtained on 5W/SBA and similar behaviours on $11 \mathrm{~W} / \mathrm{SBA}$ and 20W/SBA. However only slight differences were observed between the conversions of the $11 \mathrm{~W} / \mathrm{SBA}$ and $20 \mathrm{~W} / \mathrm{SBA}$ with values higher than $90 \%$.

\subsection{Oxidation of industrial SRGO}

Performance of the catalysts were evaluated in the oxidation of a more complex feed, a straight run gas oil with both alkyl benzothiophenes $\mathrm{C} x$-BTs (retention times below $15 \mathrm{~min}$ ) and alkyl dibenzothiophenes C $x$-DBTs (retention times higher than $15 \mathrm{~min}$ ) and containing $2000 \mathrm{ppm}$ of sulfur (SRGO2000), obtained by dilution of a real SRGO containing $1 \%$ sulfur with the previous LGO (50 ppmS). Chromatograms of the reaction medium are presented in Fig. 6 for 5W/SBA and 20W/SBA, and in Fig. SI $5 \dagger$ for $11 \mathrm{~W} / \mathrm{SBA}$. Due to the complexity of the feed, the conversion of individual molecules could not be followed. Indeed on the chromatograms after oxidation, peaks of sulfones of the C $x$-BTs appear in the zone of the C $x$-DBTs, with possible overlap of the peaks of compounds from both families. However, in the range of retention times prior to $15 \mathrm{~min}$ and
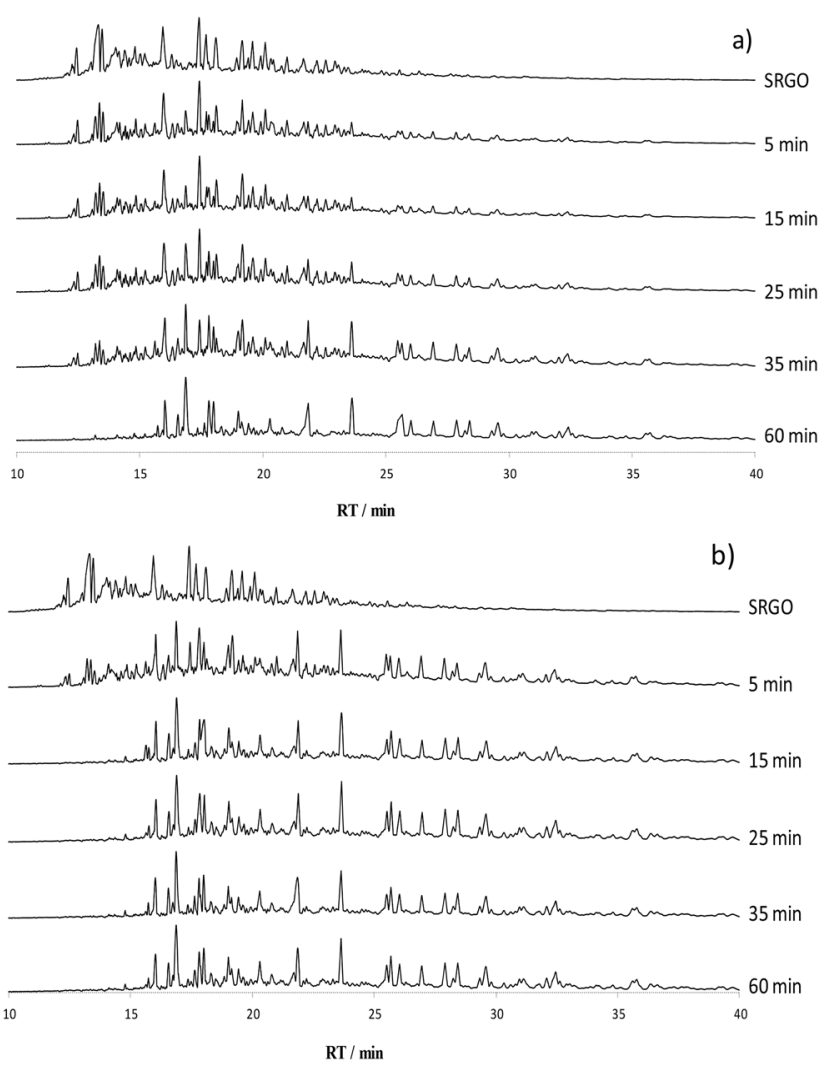

Fig. 6 Chromatograms of the reaction mixture in the ODS of SRGO 2000 on 5W/SBA (a) and 20W/SBA (b). 
after $24 \mathrm{~min}$, the conversion of the Cx-BTs and the apparition of DBT sulfones can be monitored without interaction with other compounds, since the sulfides observed at retention times superior to $24 \mathrm{~min}$ are present in very low amounts. On 5W/SBA, low intensity sulfones peaks began to appear in the region of the chromatogram with retention times higher than $24 \mathrm{~min}$ at 5 min of reaction and increased until 35 min of reaction, while BTs peaks decreased slowly and have almost disappeared at only $1 \mathrm{~h}$ of reaction. 11W/SBA and 20W/SBA appeared more efficient, sulfones being present in large quantity at $3 \mathrm{~min}$ and BTs being almost totally converted at $15 \mathrm{~min}$. The performance of $4 \mathrm{~W} / \mathrm{SiO}_{2}$ was compared to that of $5 \mathrm{~W} / \mathrm{SBA}$ with similar loading (Fig. SI6†). Unconverted BTs were still present in noticeable quantity after $2 \mathrm{~h}$ of reaction on $4 \mathrm{~W} / \mathrm{SiO}_{2}$ while they have completely disappeared on $5 \mathrm{~W} / \mathrm{SBA}$, confirming on real feedstock the better efficiency of SBA supported catalysts already evidenced on model compounds. Literature is scarce on the ODS of SRGO and 11W/SBA and 20W/SBA appear as very efficient in comparison. On a cycle oil with $3700 \mathrm{ppmS}$, with a catalyst Ti-SBA-15, TBHP $/ \mathrm{S}=2.5$, Cho et al. observed in a fixed bed reactor a total conversion of both BTs and DBTs after $48 \mathrm{~h}$ of reaction. ${ }^{32}$ Rafiee et al. performed the ODS of a crude oil with 1000 ppmS on HPW supported on montmorillonite, with $\mathrm{H}_{2} \mathrm{O}_{2}$ as oxidant and $\mathrm{O} / \mathrm{S}$ ratio of 10 and in the presence of an extractant (for $80 \mathrm{~min}$ ). The global sulfur content remaining in the reaction medium was measured and corresponded to $60 \%$ of sulfur removal. ${ }^{33}$

Retention rates were calculated on all $x \mathrm{~W} / \mathrm{SBA}$ solids after one hour of test and corresponded to $20 \%$ of the formed sulfones. Presence of sulfones in the reaction medium was found to depend on the feed: the retention rate is higher in the ODS of model solution than in the ODS of real feeds, with $98 \%$, $50 \%$ and $20 \%$ in the ODS of M500, LGO50 and SRGO2000 respectively. Solubility of sulfones is lower in apolar solvent used in model solutions than in the real feeds. Indeed the high aromatic content in the real feed may facilitate the solubility of sulfones rather than their precipitation. ${ }^{34}$

Quantitative analysis of the catalysts efficiency was performed by following the oxidation of the BTs and the formation of DBTs sulfones (Fig. 7 and SI7†). Decrease of the amount of BTs was monitored by measuring the BTs residual quantity in solution expressed in percentage of the area of the BTs peaks in the chromatogram of the initial SRGO. Increase of the amount of DBTs sulfones was monitored by measuring the DBTs sulfones quantity in solution expressed in percentage of the area of the DBTs sulfones peaks in the final chromatogram of the oxidized SRGO, taking into account that the retention rate of sulfones (around 20\% as calculated at the end of the test for each catalyst for all solids) is constant during the catalytic test.

The conversions of BTs and the formation of DBTs sulfones (in percentage in Fig. 7 and SI7 $\dagger$ ) were very similar on catalysts $11 \mathrm{~W} / \mathrm{SBA}$ and $20 \mathrm{~W} / \mathrm{SBA}$, slightly in favor of the most loaded solid, and appeared to be slower on 5W/SBA, even if at $1 \mathrm{~h}$ of reaction all solids presented almost the same efficiency. On the three catalysts, independently of their efficiency, conversion of BTs compounds and $\mathrm{DBTO}_{2}$ formation corresponding to the conversion of DBTs compounds, appeared to be simultaneous.

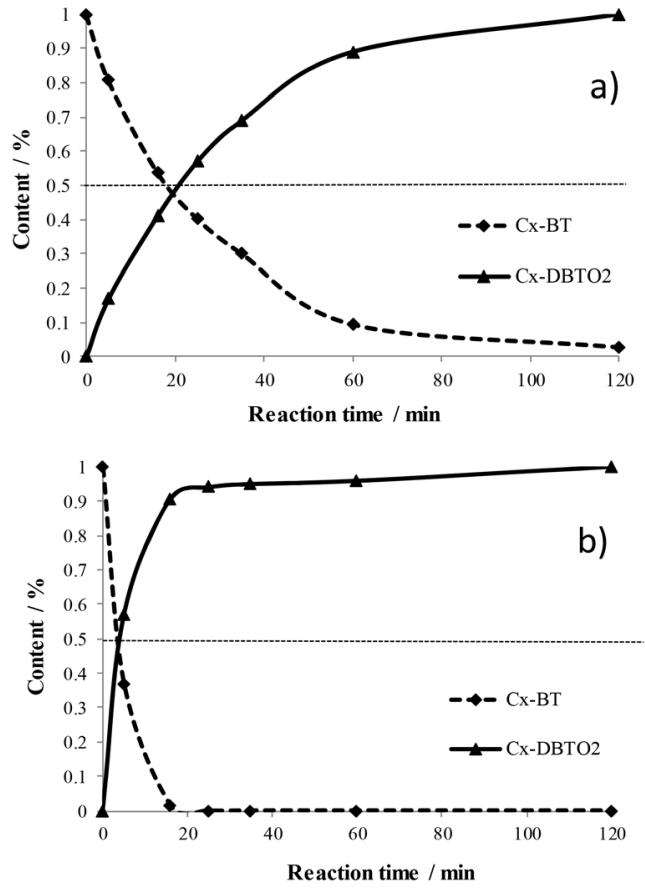

Fig. 7 Evolution of $C x$-BTs and Cx-DBT sulfones in the ODS of SRGO2000 on 5W/SBA (a) and 20W/SBA (b).

In the case of this SRGO2000, there is thus no difference in global reactivity between BTs and DBTs, which is not the case in the ODS of model feed like MM600 and MM1500. This study points out the difficulty of extrapolating results obtained on model feed to real ones.

\section{Conclusions}

In the present work, HPW/SBA based catalysts were prepared by dry impregnation of phosphotungstic acid (HPW) on SBA-15 support. On all solids the HPW heteropolycompound was preserved after calcination on all solids.

In simple model feed with only DBT and sulfur content of 50 and 500ppms, all solids $x \mathrm{~W} / \mathrm{SBA}$ were found equally performant with conversion close to $100 \%$ after one hour of test. Increasing the sulfur content to $1500 \mathrm{ppmS}$ in model feed and using LGO and SRGO allowed to discriminate the catalysts performance and 5W/SBA appeared less efficient than $11 \mathrm{~W}$ and $20 \mathrm{~W} / \mathrm{SBA}$, these two showing similar performance (at one hour of reaction). However, a monitoring of the concentration of the sulfur species during the first minutes of reaction on LGO and SRGO evidenced a significantly slower and lower oxidation on 5W/ SBA, and a slightly better performance on $20 \mathrm{~W} / \mathrm{SBA}$ than on 11W/SBA.

Owing to a careful integration of the sulfur peaks on the SCD chromatograms, reactivity of sulfide molecules was monitored in model solutions as well as in real feeds. In particular, the reactivity of a range of alkyl DBT compounds (with alkyl groups from $\mathrm{C} 2$ to $\mathrm{C} 5$ ) was followed in LGO and was found to increase in the order: 4,6-DEDBT < 4E6MDBT $\approx$ C5-DBT- $1<4,6$-DMDBT $<$ TMDBT-1 < TMDBT-2. It confirms the importance of the steric 
hindrance of alkyl substituents in the 4,6 position near the $\mathrm{S}$ atom, as well as of the size of the alkyl groups. In model feed, the reactivity of C1-BT was found much lower than that of 4,6DMDBT and DBT, while in SRGO2000, the global reactivities of BTs and DBTs compound were found similar.

Precipitation of sulfones is an important issue that needs to be addressed in ODS studies. Indeed the great majority of formed sulfones was precipitated or retained on the catalyst during ODS of model feeds (98\% of retention), while $50 \%$ in LGO and $80 \%$ in SRGO of the formed sulfones were present in the reaction medium in our conditions, in relation with the sulfones solubility in the different matrix. We also evidenced that $\mathrm{C} 1-\mathrm{BTO}_{2}$ was more soluble in the reaction medium than $\mathrm{DBTO}_{2}$ compound in the ODS of MM1500. The presence of sulfones precipitated or retained on the solids may induce deactivation of the catalysts, pointing out the importance of the textural properties of the support chosen. Indeed a catalyst prepared by impregnation of a commercial silica was found significantly less efficient than W/SBA catalyst with the same loading, the use of a mesoporous support allowing on the one hand the presence of well-dispersed tungsten species and also possibly less deactivation due to sulfone deposition during oxidation.

Moreover this study points out the difficulty to extrapolate results obtained in the ODS of model solutions to behavior in the ODS of real feeds.

\section{Conflicts of interest}

There are no conflicts to declare.

\section{Acknowledgements}

Chevreul Institute (FR 2638), Ministère de l'Enseignement Supérieur et de la Recherche, Région Nord - Pas de Calais and FEDER are acknowledged for supporting and funding partially this work.

\section{Notes and references}

1 F. Bataille, J. L. Lemberton, G. Perot, P. Leyrit, T. Cseri, N. Marchal and S. Kasztelan, Appl. Catal., A, 2001, 220, 191.

2 F. Bataille, J. L. Lemberton, P. Michaud, G. Perot, M. Vrinat, M. Lemaire, E. Schulz, M. Breysse and S. Kasztelan, J. Catal., 2000, 191, 409.

3 D. Solis, A. L. Agudo, J. Ramirez and T. Klimova, Catal. Today, 2006, 116, 469.

4 A. Stanislaus, A. Marafi and M. S. Rana, Catal. Today, 2010, 153, 1 .

5 J. T. Sampanthar, H. Xiao, J. Dou, T. Y. Nah, X. Rong and W. P. Kwan, Appl. Catal., B, 2006, 63, 85.

6 A. Ishihara, D. Wang, F. Dumeignil, H. Amano, E. W. Qian and T. Kabe, Appl. Catal., A, 2005, 279, 279.
7 M. A. Rezvani, A. F. Shojaie and M. H. Loghmani, Catal. Commun., 2012, 25, 40.

8 M. Li, M. Zhang, A. Wei, W. Zhu, S. Xun, Y. Li, H. Li and H. Li, J. Mol. Catal. A: Chem., 2015, 406, 23.

9 S. Xun, W. Zhu, F. Zhu, Y. Chang, D. Zheng, Y. Qin, M. Zhang, W. Jiang and H. Li, Chem. Eng. J., 2015, 280, 256.

10 H. Zheng, Z. Sun, X. Chen, Q. Zhao, X. Wang and Z. Jiang, Appl. Catal., A, 2013, 467, 26.

11 X. S. Wang, L. Li, J. Liang, Y.-B. Huang and R. Cao, ChemCatChem, 2017, 9, 971.

12 Y. Du, P. Yang, S. Zhou, J. Li, X. Du and J. Lei, Mater. Res. Bull., 2018, 97, 42.

13 D. Shen, Y. Dai, J. Han, L. Gan, J. Liu and M. Long, Chem. Eng. J., 2018, 332, 563.

14 D. Wang, W. Qian, H. Amano, K. Okata, A. Ishihara and T. Kabe, Appl. Catal., A, 2003, 253, 91.

15 S. Otsuki, T. Nonaka, N. Takashima, W. Qian, A. Ishihara, T. Imai and T. Kabe, Energy Fuels, 2000, 14, 1232.

16 M. A. Rezvani, M. Shaterian, F. Akbarzadeh and S. Khandan, Chem. Eng. J., 2018, 333, 537.

17 J. L. Garcia-Gutierrez, G. C. Laredo, P. Garcia-Gutierrez and F. Jimenez-Cruz, Fuel, 2014, 138, 118.

18 M. A. Safa, T. Al-Shamary, R. Al-Majren, R. Bouresli and X. Ma, Energy Fuels, 2017, 31, 7464.

19 H. A. Elwan, M. T. Zaky, A. S. Farag, F. S. Soliman and M. E. D. Hassan, J. Mol. Liq., 2017, 248, 549.

20 R. Hamiye, C. Lancelot, P. Blanchard, J. Toufaily, T. Hamieh and C. Lamonier, Fuel, 2017, 210, 666.

21 D. Zhao, Q. Huo, J. Feng, B. F. Chmelka and G. D. Stucky, J. Am. Chem. Soc., 1998, 120, 6024.

22 M. Chamack, R. Mahjoub and H. Aghayan, Chem. Eng. Res. Des., 2015, 94, 565-572.

23 Q. Gu, W. Zhu, S. Xun, Y. Chang, J. Xiong, M. Zhang, W. Jiang, F. X. Zhu and H. M. Li, Fuel, 2014, 117, 667.

24 G. Luo, L. Kang, M. Zhu and B. Dai, Fuel Process. Technol., 2014, 118, 20.

25 Y. Jia, G. Li and G. Ning, Fuel Process. Technol., 2011, 92, 106. 26 W. Kuang, A. Rives, M. Fournier and R. Hubaut, Appl. Catal., A, 2003, 250, 221.

27 R. Thouvenot, M. Fournier and C. Rocchiccioli-Deltcheff, $J$. Chem. Soc., Faraday Trans., 1991, 87(17), 2829.

28 C. Rocchiccioli-Deltcheff, M. Amirouche, M. Che, J. M. Tatibouet and M. Fournier, J. Catal., 1990, 125, 292.

29 Z. Abdalla, B. Li and A. Tufail, J. Ind. Eng. Chem., 2009, 15, 780.

30 D. Xie, Q. He, Y. Su, T. Wang, R. Xu and B. Hu, Chin. J. Catal., 2015, 36, 1205.

31 H. X. Qi, S.-R. Zhai, W. Zhang, B. Zhai and Q. D. An, Catal. Commun., 2015, 72, 53.

32 K. S. Cho and Y. K. Lee, Appl. Catal., B, 2014, 147, 35.

33 E. Rafiee, S. Sahraei and G. R. Moradi, Pet. Sci., 2016, 13, 760.

34 V. V. D. N. Prasad, K.-E. Jeong, H.-J. Chae, C.-U. Kim and S.-Y. Jeong, Catal. Commun., 2008, 9, 1966. 